\title{
Quantum-Path-Sensitive Inline XUV Interferometry
}

\author{
David T. Lloyd ${ }^{\mathrm{a}}$, Adam S. Wyatt ${ }^{\mathrm{b}, \mathrm{a}}$, Richard Chapman ${ }^{\mathrm{b}}$, Christopher Thornton ${ }^{\mathrm{b}}$, \\ Paulina Majchrzak $^{\mathbf{b}}$, Alfred Jones ${ }^{\mathbf{b}}$, Emma Springate ${ }^{\mathrm{b}} \&$ Kevin O'Keeffe $^{\mathrm{c}}$ \\ ${ }^{a}$ Clarendon Laboratory, Department of Physics, University of Oxford, Parks Road, Oxford, OX1 3PU, UK \\ ${ }^{b}$ Central Laser Facility, STFC Rutherford Appleton Laboratory, Harwell OX11 OQX, UK \\ ${ }^{c}$ Department of Physics, College of Science, Swansea University, Singleton Park, Swansea, SA2 8PP, UK \\ k.okeeffe@swansea.ac.uk
}

\begin{abstract}
An XUV interferometer composed of two inline high harmonic generation regions is used to characterize different ionized electron quantum paths. We unambiguously observe interference between long and short trajectories, revealing their attosecond-scale relative timing.
\end{abstract}

OCIS codes: $320.7100,340.7450$

High harmonic generation (HHG) is a method for producing attosecond-duration pulses in the extreme ultraviolet and soft x-ray spectral regions. For all but the highest harmonic photon energies, it is well-known that two different quantum paths may contribute, referred to as "long" and "short" due to the difference in time an electron spends away from its parent ion during the HHG process. The phase of the generated harmonics depends sensitively both on the quantum path and the intensity of the driving laser pulse, resulting in the emission of radiation which exhibits strong space-time coupling. Precise characterization of the phase contribution to the harmonic field arising from quantum paths is therefore key to fully exploiting the space-time properties of the emitted attosecond extreme ultraviolet radiation, as well as providing new insights into the ultrafast electron dynamics of the emitting species.

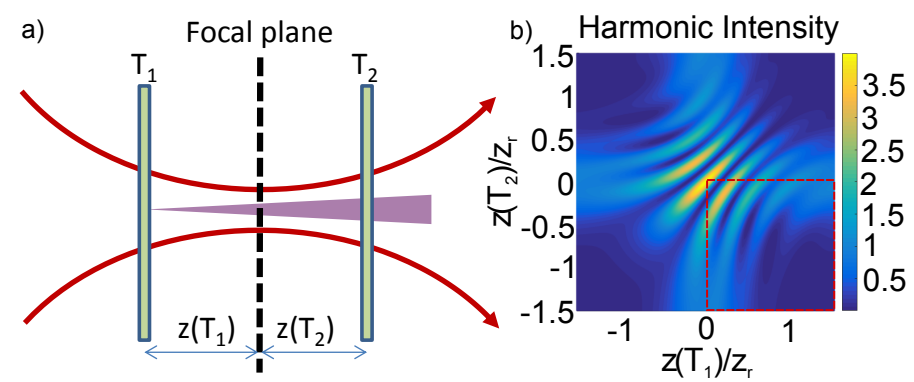

Fig. 1. a) Schematic of the XUV interferometer: thin gas cells are labelled $T_{1}$ and $T_{2}$, their relative positions were varied in order to observe interference in the measured high harmonic spectrum. b) Calculated intensity for one harmonic order as a function of gas cell locations $\left(z_{\mathrm{r}}\right.$ is the Rayleigh range), based upon a simple 1-D model of the harmonic phase variation within the focal region. The red dashed line marks the subset which could be spanned in the current experimental setup.

Compared with previous implementations of in-line XUV interferometry [1], our work sheds new light onto the fundamental quantum nature of HHG by enabling the relative phase and timing between the long and short quantum path contributions to be determined. The operation of the interferometer is shown to be robust over a broad parameter space, with interference demonstrated for harmonics driven in an elliptically polarized laser field, at long-wavelength $\left(\lambda_{0}=1100-1300 \mathrm{~nm}\right)$ fundamental driving fields, and from differing gas species.

A schematic of the experimental arrangement is found in Fig.1 a). Angularly-resolved harmonic spectra are recorded as a function of the positions of two thin gas cells within the focal region. Both cells can be moved independently allowing a two dimensional interference pattern for each harmonic order to be recorded for all divergence angles. Three such interference patterns for order $q=15$ of the $780 \mathrm{~nm}$ wavelength fundamental are presented in Fig. 2, evaluated: (d) on-axis, (e) $1.8 \mathrm{mrad}$ off-axis, and (f) $2.9 \mathrm{mrad}$ off-axis. The measured interference pattern evolves dramatically as the region of interest moves off-axis. 
To understand the evolution of the fringe pattern we extend the model of Catoire et al. [2] to two longitudinally separated generation regions. After numerically propagating the total harmonic field we evaluate the 2-D interference pattern at various divergence angles, shown in Fig. 2 g-i). Despite the simplicity of the model, the agreement between theory and experiment is striking. Our results indicate that the on-axis region is dominated by the short trajectory contribution, far off-axis the long-trajectory is dominant, and in between the two is a region where both long and short trajectory contributions have equal weight. The fringe pattern in the equal-weight region is sensitive to the relative phase between the two trajectories ( $\frac{\pi}{2}$ for the data shown), allowing us to determine this timing from the data (as a function of harmonic order, driver intensity and ellipticity).
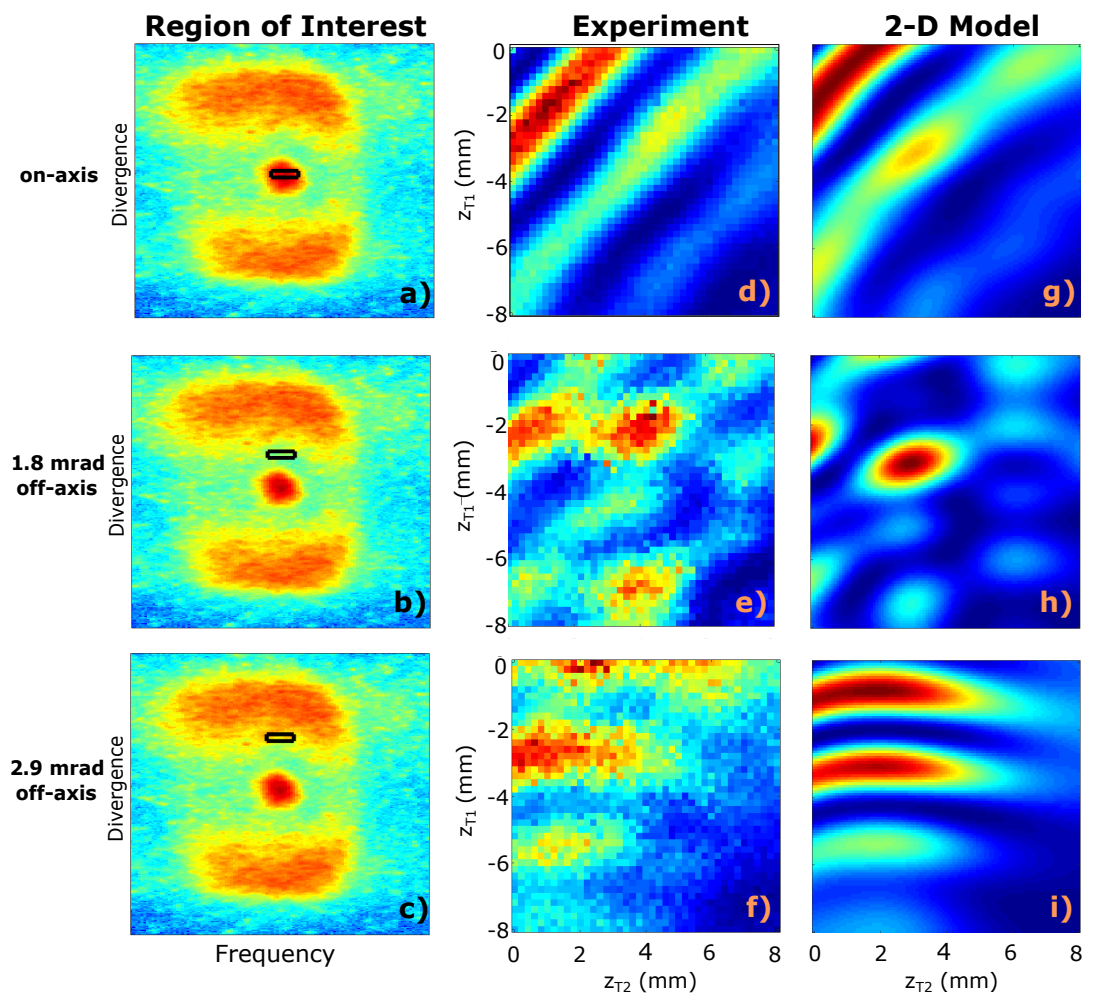

Fig. 2. Left column: Measured angularly-resolved spectrum for harmonic order $q=15$. The black box shows the region of interest selected with a) on-axis, b) $1.8 \mathrm{mrad}$ off-axis, and c) $2.9 \mathrm{mrad}$ offaxis. Middle Column: d), e), and f) show the measured 2-D interference patterns resulting from the regions of interest highlighted in a), b), and c), respectively. Right column: $\mathbf{g}), \mathbf{h}$ ), and i) show the simulated 2-D interference patterns corresponding to the regions of interest shown in a), b), and c).

We have also measured the interference patterns arising from HHG from different gas species (noble gases and molecules) and observed a clear species-dependent phase shift. This phase shift arises from the change in accumulated phase and kinetic energy of the recombining electron as a direct result of a change in the ionization potential. Whilst detailed analysis is ongoing this phase shift can be directly attributed to the different atomic/molecular dipole phases of the generating media [3], opening a route for probing strong-field phenomena in more complex systems.

Our results present a clear demonstration of quantum path interference, granting experimental access to the relative weight and phase-shifts present in the harmonic emission. The high timing stability inherent in this setup, together with quantum-path-sensitivity, may provide direct insight into complex multi-electron dynamics of molecular systems in strong laser fields with attosecond temporal resolution.

\section{References}

1. D. E. Laban et al., Physical Review Letters, 109(26), 263902 (2012)

2. F. Catoire et al., Physical Review A, 94, 063401 (2016)

3. P. Balcou, Physical Review A, 55, 3204 (1997)] 